\title{
Evaluation of Probiotic Properties of Yeasts Isolated from Sidra - An Ethnic Fermented Fish Product of North East India
}

\author{
Jasveen Bajwa and Nivedita Sharma* \\ Microbiology Research Laboratory, Department of Basic Sciences, Dr. Y S Parmar University \\ of Horticulture and Forestry, Nauni, Solan-173230, HP, India \\ *Corresponding author
}

\section{A B S T R A C T}

\begin{tabular}{|l|}
\hline K e y w o r d s \\
Sidra, Morphological, \\
Biochemical, \\
Antagonistic activity, \\
Acid and bile tolerance, \\
Hydrophobicity. \\
Autoaggregation and \\
Coaggregation
\end{tabular}

\section{Introduction}

Geographically North East (NE) India is located within the Eastern Himalayas and Purvanchal Himalayas.

The Eastern Himalayan region lies between the latitudes $26^{\circ} \quad 40^{\prime}-29^{\circ} 30^{\prime}$ North and longitudes $88^{\circ} 5^{\prime}-97^{\circ} 5^{\prime}$ East and covers a total area of 93,988 $\mathrm{km} 2$ comprising two North East states, viz. Sikkim and Arunachal Pradesh, besides eastern Nepal, Darjeeling hills in India, Bhutan and Tibetan Autonomous Region in China (Tamang, 2010). Fermented foods have heterogeneousness of traditions and cultural preferences found in the different geographical areas, where they are produced. They have been consumed since ancient times due to their prolonged shelf life, reduced volume, shorter cooking times and superior nutritive value as compared to the nonfermented ingredients. Fermentation processes are considered as developed in order to preserve food (Rolle and Satin, 2002). Ethnic fermented foods and alcoholic beverages and drinks have been consumed by the ethnic people of North East India for more than 2500 years old as per the historical records (Tamang, 2010). 
Seafood is atypical to North East India, people catch the available fishes from the various sources and preserve them traditionally (Tamang, 2010). The ethnic people use their indigenous knowledge to preserve the fish, these methods have still being used to preserve fishes which are located near water bodies with plenty of freshwater fishes. These fish products are generally consumed in the daily diet of the people.

These traditionally preserved fish are smoked and sun dried by the traditional people of North East India. Sidra is an ethnic fermented sun-dried fish product commonly consumed in the North East India, its pickle is a popular dish, the microbial composition of sidra includes bacteria (L. lactis subsp. cremoris, L. lactis subsp. lactis, L. plantarum, L. mesent eroides, E. faecium, E. faecalis, $P$. pentosaceu $s$, and Weissella confusa) and yeasts (Candida chiropterorum, C. bombicola, and Saccharomycopsis spp.). Preservation of perishable fishes by the indigenous people residing near the coastal regions is done through fermentation, sun drying, smoking, and salting without refrigeration, and are consumed as seasoning, condiments, and side dishes (Salampessy et al., 2010).

The aim of present work has been to identify the yeast species occurring during fermentation of sidra and to characterize new yeast strains with probiotic potential. The isolated yeasts strains were further tested against various food borne pathogens and their ability to survive gastrointestinal conditions such as high bile salt concentration and low $\mathrm{pH}$. Furthermore their probiotic attributes were also assessed.

\section{Materials and Methods}

The current research was divided in some steps, as follows: a) yeast isolation from fermented fish product sidra; b) phenotypic and genotypic characterization; c) Analysis to select the most promising strains, by assessing their probiotic attributes i.e., acid and bile tolerance, autoaggregation and coaggregation, hydrophobicity.

All the chemicals used in the study were purchased from Hi-Media.

\section{Yeast isolation}

In total, 31 yeast isolates were isolated from sidra. The fermentation of sidra was conducted by the indigenous people household scale.1gm of sample was diluted with $9 \mathrm{ml}$ of a sterile saline solution $(0.9 \% \mathrm{NaCl})$ and homogenized for $60 \mathrm{~s}$. The homogenates were serially diluted in saline solution and plated onto the Yeast Universal Medium (YM) (Yeast Extract, $3.0 \mathrm{~g} / \mathrm{L}$; Malt Extract, $3.0 \mathrm{~g} / \mathrm{L}$; Peptone, $5.0 \mathrm{~g} / \mathrm{L}$; Glucose, $10.0 \mathrm{~g} / \mathrm{L}$; agar, 15 $\mathrm{g} / \mathrm{L}$ ), incubated at $25^{\circ} \mathrm{C}$ for $2-4$ days for the evaluation of yeasts. Plates were incubated for $72 \mathrm{~h}$ at $28{ }^{\circ} \mathrm{C}$. Yeasts, showing the typical appearance of Saccharomyces and Candida (white-to-yellow colonies) and the typical cell appearance at the microscope, were randomly selected and labeled with code names. The selected yeast strains were further purified by successive streaking on YM media. All isolates were maintained at $-80{ }^{\circ} \mathrm{C}$ in $20 \%$ (v/v) glycerol (Hi-Media). Isolates were propagated in YM broth

\section{Identification of sidra yeast isolates to species level by genotypic and phenotypic tests}

\section{Phenotypic and biochemical characterization}

All the selected yeast isolates were phenotypically characterized according to Kurtzman et al., (2011b), and further biochemical tests were carried out i.e catalase reaction, carbohydrate fermentation, hydrogen 
sulphide formation, urease activity and growth at different temperature

\section{Biochemical characterization}

\section{Catalase activity}

Catalase activity of yeasts was evaluated by adding $3 \%(\mathrm{v} / \mathrm{v})$ of hydrogen peroxide onto the cultured colonies, according to the Whittenbury method (1964).

The results were expressed as positive (+) or negative (-).

\section{Carbohydrate fermentation}

Carbohydrate fermentation was performed for the yeasts isolates following the method of Aneja (2003).

\section{Hydrogen sulphide production}

Hydrogen sulphide production was evaluated following the method of Aneja (2003).

\section{Urease activity}

This test was performed in the Christensen's urea agar (Oxoid), containing phenol red as $\mathrm{pH}$ indicator. After yeast inoculum, plates were incubated at $25^{\circ} \mathrm{C}$ for 2-7 days; color turning to purple highlighted urea hydrolysis and $\mathrm{pH}$ increase.

\section{Growth at different temperature}

Effect of different temperature on viability of yeasts isolates was examined by inoculating them in YEDA (yeast extract dextrose agar) broth, and incubating at 20,25,30, 35, 40 and $45^{\circ} \mathrm{C}$.

After the incubation period of $24 \mathrm{~h}$, growth was examined for thermo-tolerance of the isolates.

\section{Genotypic tests}

Isolation of genomic yeast DNA was performed according to the method described by Cocolin et al., (2000).

\section{Primary screening of probiotic properties of yeasts}

\section{Tolerance to low $\mathrm{pH}$}

The survival studies under gastrointestinal conditions were determined following the method of Conway et al., 1987

\section{Bile salt tolerance}

Tolerance to bile salt of the yeast isolates was determined following the method of Walker and Gilliland, 1993.

\section{Antimicrobial activity}

The antimicrobial activity was assessed by bit/disk method.

Spoilage causing microorganisms i.e viz Staphylococcus aureus IGMC, Leuconostoc mesenteroides MTCC 107,

Bacillus cereus CRI, Pectobacterium carotovorum MTCC 1428, Escherichia coli IGMC, Pseudomonas syringae IGMC Enterococcus faecalis MTCC 2729, Listeria monocytogens MTCC 839 and Clostridium perfringens MTCC 1739, were used to check antagonistic activity of the yeast isolates.

Secondary screening of probiotic yeast isolates

Cell surface hydrophobicity (Mishra and Prasad, 2005)

Assessment of hydrophobicity of cell surface was done based on MATS (Microbial 
Adhesion to Solvent), following the method of Mishra and Prasad, 2005.

Cell surface hydrophobicity was calculated following the equation:

$$
\underset{\%}{\text { Hydrophobicity }}=\frac{\left[\left(\mathrm{A}-\mathrm{A}_{0}\right)\right]}{\mathrm{A}} \times 100
$$

Where $\mathrm{A}$ and $\mathrm{A}_{0}$, were the absorbance before and after hydrocarbons (xylene, ethyl acetate and chloroform) extraction respectively.

Autoaggregation (Del Re et al., 2000)

Autoaggregation assay was performed as described by Del Re et al., (2000)

$$
\underset{\%}{\text { Autoaggregation }}=\frac{1-\mathrm{A}_{\mathrm{t}}}{\mathrm{A}_{0}} \times 100
$$

Where $A_{t}$ represents the absorbance at time $\mathrm{t}=1,2,3,4,5 \mathrm{~h}$ and

$\mathrm{A}_{0}$ the absorbance at $\mathrm{t}=0 \mathrm{~h}$ (i.e. 0.5 )

\section{Co-aggregation (Del Re et al., 2000)}

Coaggregation ability of each isolate was determined by following the method described by Del Re et al., (2000)

Co-aggregation $\%$ was calculated

$$
\begin{aligned}
& \text { [APath+ALAB/2] -AMix } \\
& \text { Coaggregation }(\%)=\text {------------------- } \times 100 \\
& \text { [APath-ALAB/2] }
\end{aligned}
$$

\section{Estimation of Siderophore production}

Screened probiotic isolates were checked for siderophore production by following the method of Schwyn and Neilands (1987).
Estimation of Exopolysaccharide (EPS) production

Exopolysaccharide (EPS) production was evaluated as described by Mora et al., (2002).

Estimation of $\beta$-Galactosidase production (Lin et al., 1989)

$\beta$-galactosidase activity was evaluated following the method of Lin et al., 1989

Safety profile of yeast isolates

\section{Antibiotic-resistance}

Antibiotic-resistance was carried out with the KirbyeBauer method according to the NCCLS protocol (National Committee for Clinical Laboratory Standard, 1993). The antibiotics used were, Tetracycline (30 $\mu \mathrm{g})$, Ciprofloxacin $(10 \mu \mathrm{g})$, Penicillin-G $(10 \mu \mathrm{g})$, Gentamycin $(10 \mu \mathrm{g})$, Ofloxacin $(10 \mu \mathrm{g})$, Erythromycin $(15 \mu \mathrm{g})$, Clindamycin $(10 \mu \mathrm{g})$, Fluconazole $(25 \mu \mathrm{g})$ and Ketoconazole (10 $\mu \mathrm{g})$

Haemolytic activity (Marakoudakis et al., 2009)

The haemolytic activities of potential probiotic isolates were determined according to the method of Marakoudakis et al., (2009)

DNase production (Gupta and Malik, 2007)

DNase enzyme production of isolates was determined by following the method of Gupta and Malik (2007).

Gelatinase production (Harrigan and McCance, 1990)

Gelatinase enzyme production of isolates was determined by following the method of Harrigan and McCane (1990). 


\section{Results and Discussion}

\section{Phenotypic and characterization}

Physiological and Biochemical characterization of the yeasts isolates had been done and their characteristics were noted down in Table 1-3.

Pedersen et al., (2012), reported yeast isolates from Fura, a spontaneously fermented pearl millet product consumed in West Africa, and these were identified by pheno- and genotypic methods. Perricone (2014), isolated probiotic yeasts from cereal based food and beverages following technological and biochemical characterization.

\section{Antimicrobial activity}

Probiotic yeast strains isolated from sidra were tested for their antagonistic activity against selected food borne/spoilage causing microorganisms viz. Staphylococcus aureus IGMC; Leuconostoc mesenteroides MTCC 107; Bacillus cereus CRI; Pectobacterium carotovorum MTCC 1428; Escherichia coli IGMC; Pseudomonas syringae IGMC; Enterococcus faecalis MTCC 2729; Listeria monocytogens MTCC 839; Clostridium perfringens MTCC 1739. The data on inhibitory spectrum of probiotic yeast by bit/disc method had been shown in Table 4 .

\section{Genotypic tests}

The best selected yeast isolates on the basis of their broadest antagonism against pathogenic bacteria were identified at genomic level by using ITS gene technique. Genomic DNA of two best yeast isolates was isolated by following the method of Hashem et al., (2010).

\section{Primary screening of probiotic properties of yeasts}

\section{Tolerance to low pH}

To resist acidic $\mathrm{pH}$ and remain viable under gastric juices is an important characteristic of probiotic microorganisms. Acid tolerance of the screened potential probiotic isolates was studied by suspending bacterial cells in phosphate buffer saline of different $\mathrm{pH}$ 1.0, 2.0 and 3.0 followed by their incubation for 0 , 60,120 and $180 \mathrm{~min}$.

It was observed in this experiment that cells of all the isolates could tolerate an incubation of 60 to $120 \mathrm{~min}$ at $\mathrm{pH} 1.0$ to 3.0. (Table 5).

Fig.1 Neighbor-joining with 1000 bootstrap values in MEGA 6.0 showing phylogenetic relationship of Debaryomyces hansenii F3 based on a distance matrix analysis of

$16 \mathrm{~S}$ rRNA sequences

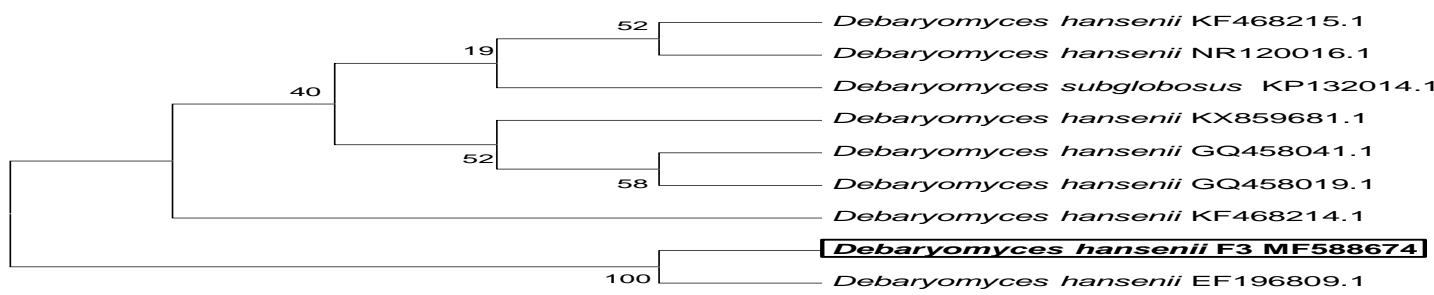


Fig.2 Neighbor-joining with 1000 bootstrap values in MEGA 6.0 showing phylogenetic relationship of Candida psychrophila F6 based on a distance matrix analysis of 16S rRNA sequences

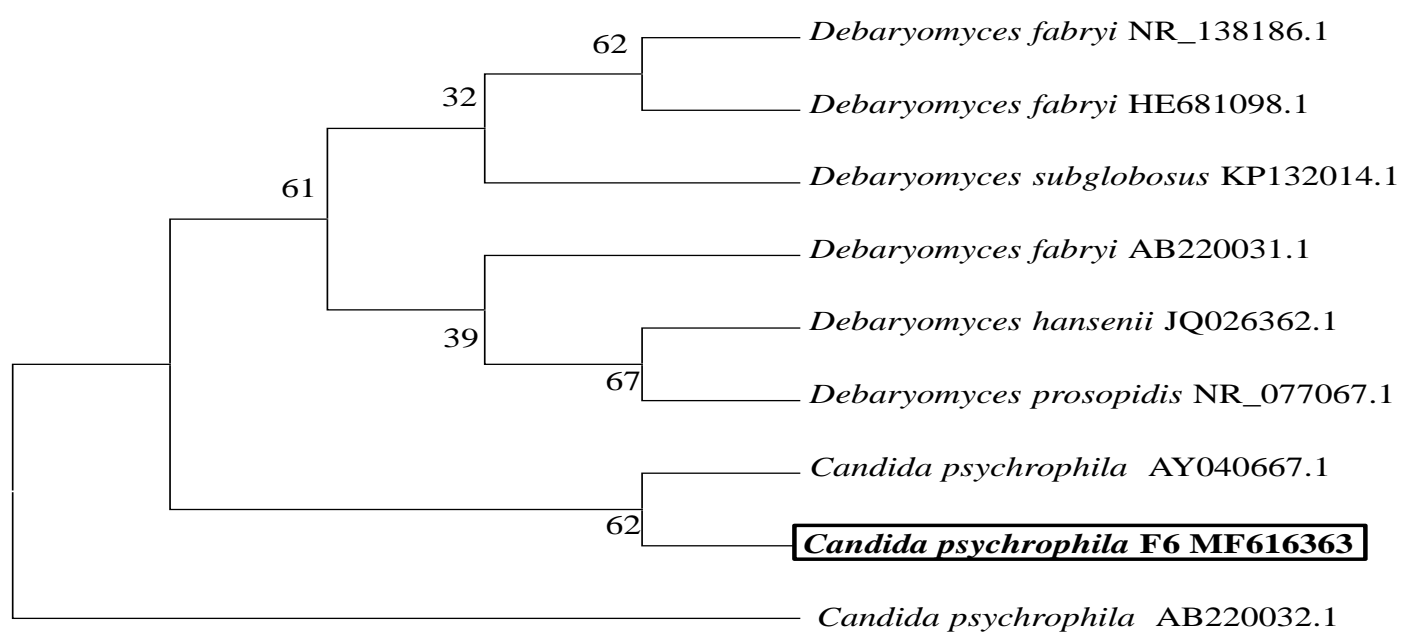

Table.1 Morphological characteristics of probiotic yeasts isolates

\begin{tabular}{|l|l|l|l|l|l|l|l|}
\hline S. No & Isolate & Source & Color & Surface & Margin & Elevation & Cell-shape \\
\hline $\mathbf{1}$ & YS & Sidra & Cream & Round & Undulate & Convex & Round \\
\hline $\mathbf{2}$ & YS1 & Sidra & Cream & Round & Undulate & Convex & Ellipsoid \\
\hline $\mathbf{3}$ & Y5 & Sidra & Cream & Round & Entire & Convex & Oval \\
\hline $\mathbf{4}$ & Y5(A) & Sidra & Cream & Round & Entire & Raised & Ellipsoid \\
\hline $\mathbf{5}$ & Y4 & Sidra & Cream & Smooth & Entire & Raised & Ellipsoid \\
\hline $\mathbf{6}$ & F1 & Sidra & Cream & Wrinkled & Entire & Convex & Rough \\
\hline $\mathbf{7}$ & F2 & Sidra & White & Smooth & Undulate & Convex & Rough \\
\hline $\mathbf{8}$ & F3 & Sidra & White & Rough & Undulate & Raised & Ellipsoid \\
\hline $\mathbf{9}$ & F4 & Sidra & Yellow & Rough & Etire & Raised & Ellipsoid \\
\hline $\mathbf{1 0}$ & F5 & Sidra & Yellow & Smooth & Entire & Convex & Rough \\
\hline $\mathbf{1 1}$ & F6 & Sidra & Cream & Wrinkled & Entire & Flat & Ellipsoid \\
\hline $\mathbf{1 2}$ & F7 & Sidra & Cream & Wrinkled & Entire & Raised & Ellipsoid \\
\hline 13. & F8 & Sidra & Yellow & Rough & Undulate & Convex & Ellipsoid \\
\hline
\end{tabular}


Table.2 Biochemical characteristics of probiotic yeasts isolates

\begin{tabular}{|c|c|c|c|c|c|}
\hline Sr. No. & Isolate & Catalase test & $\begin{array}{l}\text { Carbohydrate } \\
\text { utilization }\end{array}$ & $\mathrm{H}_{2} \mathrm{~S}$ production & Urease test \\
\hline 1. & YS & -ve & $\mathrm{AG}^{-}$ & $-\mathrm{ve}$ & $-\mathrm{ve}$ \\
\hline 2. & YS1 & $+\mathrm{ve}$ & AG & $-\mathrm{ve}$ & $-\mathrm{ve}$ \\
\hline 3. & Y5 & -ve & $\mathrm{AG}^{-}$ & $-\mathrm{ve}$ & -ve \\
\hline 4. & Y5(A) & -ve & $A G$ & $-\mathrm{ve}$ & $-\mathrm{ve}$ \\
\hline 5. & Y4 & -ve & $\mathrm{AG}^{-}$ & $-v e$ & $-v e$ \\
\hline 6. & $\mathrm{~F} 1$ & $+\mathrm{ve}$ & $\mathrm{AG}^{-}$ & $-v e$ & -ve \\
\hline 7. & $\mathrm{~F} 2$ & $+\mathrm{ve}$ & $\mathrm{AG}$ & $-\mathrm{ve}$ & $-\mathrm{ve}$ \\
\hline 8. & F3 & -ve & $\mathrm{AG}^{-}$ & $-\mathrm{ve}$ & $-\mathrm{ve}$ \\
\hline 9. & $\mathrm{~F} 4$ & -ve & AG & $-\mathrm{ve}$ & $-\mathrm{ve}$ \\
\hline 10. & F5 & $+\mathrm{ve}$ & $\mathrm{AG}^{-}$ & $-\mathrm{ve}$ & $-\mathrm{ve}$ \\
\hline 11. & F6 & -ve & $\mathrm{AG}^{-}$ & $-\mathrm{ve}$ & $-\mathrm{ve}$ \\
\hline 12. & F7 & $+\mathrm{ve}$ & AG & $-v e$ & $-v e$ \\
\hline 13. & F8 & $+\mathrm{ve}$ & $\mathrm{AG}^{-}$ & $-\mathrm{ve}$ & -ve \\
\hline
\end{tabular}

Table.3 Growth characteristics of probiotic yeasts isolates from traditional fermented food matrices of North-East India

\begin{tabular}{|c|c|c|c|c|c|c|}
\hline \multirow[t]{2}{*}{ S.No } & \multirow[t]{2}{*}{ Isolate } & \multicolumn{5}{|c|}{ Growth temperature } \\
\hline & & $25^{\circ} \mathrm{C}$ & $30^{\circ} \mathrm{C}$ & $35^{\circ} \mathrm{C}$ & $37^{\circ} \mathrm{C}$ & $40^{\circ} \mathrm{C}$ \\
\hline 1. & YU & + & + & + & - & - \\
\hline 2. & YU1 & + & + & + & - & - \\
\hline 3. & YU2 & + & + & + & - & - \\
\hline 4. & U1 & + & + & + & - & - \\
\hline 5. & U2 & + & + & + & - & - \\
\hline 6. & YS & + & + & + & + & - \\
\hline 7. & YS1 & + & - & - & - & - \\
\hline 8. & Y5 & + & + & + & + & - \\
\hline 9. & Y5(A) & + & + & + & - & - \\
\hline 10. & $\mathrm{Y} 4$ & + & - & - & - & - \\
\hline 11. & F1 & + & + & + & + & - \\
\hline 12. & F2 & + & + & + & - & - \\
\hline 13. & F3 & + & + & + & - & - \\
\hline 14. & $\mathrm{~F} 4$ & + & - & - & - & - \\
\hline 15. & F5 & + & + & + & + & - \\
\hline 16. & F6 & + & + & + & + & - \\
\hline 17. & F7 & + & + & + & - & - \\
\hline 18. & F8 & + & + & + & + & - \\
\hline
\end{tabular}


Table.4 Preliminary screening of probiotic yeasts isolated traditional fermented food matrices of North-East India on the basis of their antagonistic pattern against test indicators by bit/disc method

\begin{tabular}{|c|c|c|c|c|c|c|c|c|c|c|c|c|c|}
\hline $\begin{array}{l}\text { Sr. } \\
\text { No. }\end{array}$ & $\begin{array}{l}\text { Name } \\
\text { of } \\
\text { isolate }\end{array}$ & Source & $\begin{array}{l}\text { S. } \\
\text { aureus } \\
(\mathrm{mm})\end{array}$ & $\begin{array}{l}\text { L. } \\
\text { mesenteroides } \\
(\mathrm{mm})\end{array}$ & $\begin{array}{l}\text { B. } \\
\text { cereus }(\mathrm{mm})\end{array}$ & $\begin{array}{l}P . \\
\text { carotovarum } \\
(\mathrm{mm})\end{array}$ & $\begin{array}{l}E . \\
\text { coli } \\
(\mathrm{mm})\end{array}$ & $\begin{array}{l}P . \\
\text { syringia } \\
(\mathrm{mm})\end{array}$ & $\begin{array}{l}E . \\
\text { feacalis } \\
(\mathrm{mm})\end{array}$ & $\begin{array}{l}\text { L. } \\
\text { monocytogens } \\
(\mathrm{mm})\end{array}$ & $\begin{array}{l}\text { C. } \\
\text { perfringenes } \\
(\mathrm{mm})\end{array}$ & Mean & $\begin{array}{l}\text { Percent } \\
\text { Inhibition } \\
(\%)\end{array}$ \\
\hline 1. & YS & Sidra & - & - & - & - & - & - & - & - & - & - & - \\
\hline 2. & YS1 & Sidra & - & - & - & - & - & - & - & - & - & - & - \\
\hline 3. & Y5 & Sidra & - & - & 9 & - & - & - & - & - & - & 1 & $11.11 \%$ \\
\hline 4. & Y5(A) & Sidra & - & - & - & - & - & - & - & - & - & - & - \\
\hline 5. & Y4 & Sidra & 14 & - & - & - & - & 9 & - & - & - & 2.55 & $22.22 \%$ \\
\hline 6. & F1 & Sidra & 12 & - & - & - & - & 10 & - & - & - & 2.44 & $22.22 \%$ \\
\hline 7. & F2 & Sidra & - & - & - & - & - & - & - & - & - & - & - \\
\hline 8. & F3 & Sidra & 15 & 10 & - & - & - & 20 & 17 & 15 & 16 & 10.33 & 66.66 \\
\hline 9. & F4 & Sidra & 9 & - & - & 9 & 18 & 9 & - & - & - & 5 & $44.44 \%$ \\
\hline 10. & F5 & Sidra & 9 & - & - & - & 17 & - & - & - & - & 2.88 & $22.22 \%$ \\
\hline 11. & F6 & Sidra & 17 & 18 & 11 & 20 & - & - & 15 & 18 & 20 & 13.22 & 77.77 \\
\hline 12. & F7 & Sidra & - & - & - & - & - & - & - & - & - & - & - \\
\hline 13. & F8 & Sidra & - & - & - & - & - & - & - & - & - & - & - \\
\hline
\end{tabular}


Table.5 Acidity tolerance of screened potential probiotic isolates

\section{Debaryomyces hansenii F3}

\begin{tabular}{|c|c|c|c|c|c|c|c|c|c|}
\hline \multirow[t]{3}{*}{ pH } & \multicolumn{9}{|c|}{ Incubation time (h) } \\
\hline & \multicolumn{5}{|c|}{ Cell survival (log CFU/ml)* } & \multicolumn{4}{|c|}{$* * \%$ Cell survival } \\
\hline & $\mathbf{0}$ & 60 & 120 & 180 & Mean & 60 & 120 & 180 & Mean \\
\hline 1.0 & 0.00 & 0.00 & 0.00 & 0.00 & 0.00 & $0.00(0.00)$ & $0.00(0.00)$ & $0.00(0.00)$ & $0.00(0.00)$ \\
\hline 2.0 & 6.23 & 4.07 & 0.00 & 0.00 & 2.57 & $\begin{array}{l}44.67 \\
(41.94)\end{array}$ & $0.00(0.00)$ & $0.00(0.00)$ & $\begin{array}{l}14.89 \\
(13.98)\end{array}$ \\
\hline 3.0 & 7.30 & 5.62 & 5.00 & 5.00 & 5.73 & $\begin{array}{l}61.69 \\
(51.76)\end{array}$ & $\begin{array}{l}54.94 \\
(47.83)\end{array}$ & $\begin{array}{l}55.55 \\
(48.18)\end{array}$ & $\begin{array}{l}57.39 \\
(49.25)\end{array}$ \\
\hline Control & 9.23 & 9.11 & 9.10 & 9.00 & 9.11 & $\begin{array}{l}100 \\
(90)\end{array}$ & $\begin{array}{l}100 \\
(90)\end{array}$ & $\begin{array}{l}100 \\
(90)\end{array}$ & $\begin{array}{l}100 \\
(90)\end{array}$ \\
\hline Mean & 5.69 & 4.7 & 3.52 & 3.5 & & 51.59 & 38.73 & 38.88 & \\
\hline $\mathrm{CD}_{0.05}$ & \multicolumn{5}{|c|}{$\begin{array}{l}\text { Treatment }(0.09) \\
\text { Incubation Time }(0.09) \\
\text { TxI }(0.17)\end{array}$} & \multicolumn{4}{|c|}{$\begin{array}{l}\text { Treatment }(0.05) \\
\text { Incubation Time }(0.06) \\
\text { TxI }(0.10)\end{array}$} \\
\hline
\end{tabular}

$* \log$ CFU/ml: Mean of results from three separate experiments

$* * \%$ Survivability $=\left(\log \mathrm{cfu} \mathrm{pH}_{1.2 .3 /} \log \mathrm{cfu} \mathrm{pH}_{.65}\right) \times 100$

\# Transformed values (Arcsign transformation)

\section{Candida pyschrophila F6}

\begin{tabular}{|c|c|c|c|c|c|c|c|c|c|}
\hline \multirow[t]{3}{*}{ pH } & \multicolumn{9}{|c|}{ Incubation time (h) } \\
\hline & \multicolumn{5}{|c|}{ Cell survival (log CFU/ml)* } & \multicolumn{4}{|c|}{$* * \%$ Cell survival } \\
\hline & 0 & 60 & 120 & 180 & Mean & 60 & 120 & 180 & Mean \\
\hline 1.0 & 8.19 & 4.94 & $\mathbf{0 . 0 0}$ & 0.00 & 3.28 & $\begin{array}{l}50.40 \\
(45.22)\end{array}$ & $0.00(0.00)$ & $0.00(0.00)$ & $\begin{array}{l}16.8 \\
(15.07)\end{array}$ \\
\hline 2.0 & 8.23 & 7.07 & 4.0 & 0.00 & 4.82 & $\begin{array}{l}72.14 \\
(58.14)\end{array}$ & $\begin{array}{l}45.66 \\
(42.51)\end{array}$ & $0.00(0.00)$ & $\begin{array}{l}87.36 \\
(33.55\end{array}$ \\
\hline 3.0 & 8.30 & 7.11 & $\mathbf{7 . 0 0}$ & 0.00 & 5.60 & $\begin{array}{l}72.55 \\
(58.40)\end{array}$ & $\begin{array}{l}79.90 \\
(63.36)\end{array}$ & $0.00(0.00)$ & $\begin{array}{l}99.18 \\
(40.58)\end{array}$ \\
\hline Control & 9.81 & 9.80 & 8.76 & 8.55 & 9.23 & $\begin{array}{l}100 \\
(90)\end{array}$ & $\begin{array}{l}100 \\
(90)\end{array}$ & $\begin{array}{l}100 \\
(90)\end{array}$ & $\begin{array}{l}100 \\
(90)\end{array}$ \\
\hline Mean & 8.63 & 7.23 & 4.94 & 2.13 & & 73.77 & 56.39 & 25 & \\
\hline $\mathrm{CD}_{0.05}$ & \multicolumn{5}{|c|}{$\begin{array}{l}\text { Treatment }(0.09) \\
\text { Incubation Time }(0.09) \\
\text { TxI }(0.18)\end{array}$} & \multicolumn{4}{|c|}{$\begin{array}{l}\text { Treatment }(0.05) \\
\text { Incubation Time }(0.06) \\
\text { TxI }(0.10)\end{array}$} \\
\hline
\end{tabular}

$* \log$ CFU/ml: Mean of results from three separate experiments

$* * \%$ Survivability $=\left(\log \mathrm{cfu} \mathrm{pH}_{1.2 .3 /} \log \mathrm{cfu} \mathrm{pH}_{.65}\right) \times 100$

\# Transformed values (Arcsign transformation)

Table.6 Bile salt tolerance of potential probiotic microorganisms

\section{Debaryomyces hensanii F3}

\begin{tabular}{|c|c|c|c|c|c|c|c|}
\hline \multirow{3}{*}{$\begin{array}{l}\text { Bile salt } \\
\text { concentration }\end{array}$} & \multicolumn{7}{|c|}{ Incubation time } \\
\hline & \multicolumn{4}{|c|}{ Cell survival $(\log \mathrm{CFU} / \mathrm{ml})^{*}$} & \multicolumn{3}{|c|}{ Cell survival $(\%)^{* *}$} \\
\hline & $\mathbf{0}$ & 4 & 8 & Mean & 4 & 8 & Mean \\
\hline 0.3 & 6.66 & 6.64 & 6.29 & 6.53 & $72.01(58.05)$ & $68.44(55.82)$ & 70.22 \\
\hline 0.5 & 6.20 & 6.19 & 6.15 & 6.18 & $67.13(55.01)$ & $66.92(54.88)$ & 67.02 \\
\hline 1.0 & 6.09 & 6.06 & 6.00 & 6.05 & $65.72(54.16)$ & $65.28(53.89)$ & 65.5 \\
\hline 1.5 & 6.00 & 6.00 & 0 & 4 & 65.07 (53.77) & $0.00(0.00)$ & 32.53 \\
\hline 2.0 & 0 & 0 & 0 & 0.00 & $0.00(0.00)$ & $0.00(0.00)$ & 0.00 \\
\hline Control & 9.45 & 9.22 & 9.19 & 9.28 & $100(90)$ & $100(90)$ & 100 \\
\hline Mean & 5.73 & 5.68 & 4.60 & & $61.65(51.83)$ & $50.10(42.43)$ & \\
\hline $\mathrm{CD}_{0.05}$ & \multicolumn{4}{|c|}{$\begin{array}{l}\text { Treatment }(0.09) \\
\text { Incubation Time }(0.12) \\
\text { TxI }(0.21)\end{array}$} & \multicolumn{3}{|c|}{$\begin{array}{l}\text { Treatment }(0.05) \\
\text { Incubation Time }(0.08) \\
\text { TxI }(0.12)\end{array}$} \\
\hline
\end{tabular}

*Log cfu/ml: Mean of results from three separate experiments

$* * \%$ Survivability $=(\log \mathrm{cfu} / \mathrm{ml} 0.3,1,2 \%$ bile salt $/ \log \mathrm{cfu} / \mathrm{ml} 10 \%$ bile salt $) \times 100$

\#Transformed values (Square root transformation) 


\section{Candida psychrophila F6}

\begin{tabular}{|c|c|c|c|c|c|c|c|}
\hline \multirow{3}{*}{$\begin{array}{l}\text { Bile salt } \\
\text { concentration }\end{array}$} & \multicolumn{7}{|c|}{ Incubation time } \\
\hline & \multicolumn{4}{|c|}{ Cell survival $(\log \mathrm{CFU} / \mathrm{ml})^{*}$} & \multicolumn{3}{|c|}{ Cell survival $(\%)^{* *}$} \\
\hline & 0 & 4 & 8 & Mean & 4 & 8 & Mean \\
\hline 0.3 & 8.22 & 8.12 & 8.12 & 8.15 & $79.60(63.14)$ & $80.07(63.48)$ & $79.83(63.31)$ \\
\hline 0.5 & 8.05 & 8.00 & 8.00 & 8.00 & $78.43(62.32)$ & $78.89(62.64)$ & $78.66(62.48)$ \\
\hline 1.0 & 8.03 & 8.00 & 8.01 & 8.01 & $78.43(62.32)$ & $78.99(62.71)$ & $78.71(62.51)$ \\
\hline 1.5 & 7.56 & 7.55 & 7.44 & 7.51 & $74.01(59.34)$ & $73.37(58.93)$ & $73.69(59.13)$ \\
\hline 2.0 & 7.27 & 7.22 & 7.00 & 7.16 & $70.78(57.27)$ & $69.03(56.18)$ & $69.90(56.72)$ \\
\hline Control & 10.34 & 10.20 & 10.14 & 10.22 & $100(90)$ & $100(90)$ & $100(90)$ \\
\hline Mean & 8.24 & 8.18 & 8.11 & & $80.20(65.73)$ & $80.05(65.65)$ & \\
\hline $\mathrm{CD}_{0.05}$ & \multicolumn{4}{|c|}{$\begin{array}{l}\text { Treatment (N/A) } \\
\text { Incubation Time }(0.16) \\
\text { TxI (N/A) }\end{array}$} & \multicolumn{3}{|c|}{$\begin{array}{l}\text { Treatment }(\mathrm{N} / \mathrm{A}) \\
\text { Incubation Time }(0.12) \\
\text { TxI }(0.18)\end{array}$} \\
\hline
\end{tabular}

$* \log \mathrm{cfu} / \mathrm{ml}$ : Mean of results from three separate experiments

$* * \%$ Survivability $=(\log \mathrm{cfu} / \mathrm{ml} 0.3,1,2 \%$ bile salt $/ \log \mathrm{cfu} / \mathrm{ml} 0 \%$ bile salt $) \times 100$

"Transformed values (Square root transformation)

Table.7 Adhesion of probiotic microorganisms to different hydrocarbons

\begin{tabular}{|c|c|c|c|c|c|c|c|c|}
\hline \multirow[t]{2}{*}{ Isolates } & \multicolumn{4}{|l|}{$\mathbf{O D}_{600}^{*}$} & \multicolumn{4}{|c|}{$\%$ Hydrophobicity** } \\
\hline & Xylene & $\begin{array}{l}\text { Chlorofo } \\
\text { rm }\end{array}$ & $\begin{array}{l}\text { Ethyl } \\
\text { acetat } \\
\text { e }\end{array}$ & Mean & $\begin{array}{l}\text { Xylen } \\
\text { e }\end{array}$ & $\begin{array}{l}\text { Chlorofo } \\
\text { rm }\end{array}$ & $\begin{array}{l}\text { Ethyl } \\
\text { acetate }\end{array}$ & Mean \\
\hline D. hansenii F3 & 0.22 & 0.22 & 0.13 & 0.19 & 78 & 78 & 87 & 81 \\
\hline C. psychrophila F6 & 0.19 & 0.27 & 0.22 & 0.22 & 81 & 73 & 78 & 77.33 \\
\hline Mean & 0.12 & 0.29 & 0.20 & & 88 & 70.11 & 79.22 & \\
\hline $\mathrm{CD}_{0.05}$ & \multicolumn{4}{|c|}{$\begin{array}{l}\mathrm{T}(0.06) \\
\mathrm{I}(0.10) \\
\mathrm{TxI}(0.18\end{array}$} & \multicolumn{4}{|c|}{$\begin{array}{l}\mathrm{T}(0.79) \\
\mathrm{I}(1.36) \\
\mathrm{TxI}(2.35)\end{array}$} \\
\hline
\end{tabular}

*OD: Mean of results from three different experiments

$* *$ Hydrophobicity $\%=\left[\left(\mathrm{A}-\mathrm{A}_{0}\right) / \mathrm{A}\right] \times 100$

Table.8 Estimation of autoaggregation potential of screened probiotic isolates

\begin{tabular}{|c|c|c|c|c|c|c|c|c|c|c|c|c|}
\hline \multirow[t]{2}{*}{ Isolates } & \multicolumn{6}{|c|}{$\mathrm{OD}_{600}{ }^{\#}$} & \multicolumn{6}{|c|}{ **Autoaggregation (\%) } \\
\hline & $1 \mathrm{~h}$ & $2 \mathrm{~h}$ & $3 h$ & $4 h$ & $5 h$ & $\begin{array}{c}\text { Mea } \\
\mathbf{n}\end{array}$ & $1 \mathrm{~h}$ & $2 \mathrm{~h}$ & $3 \mathbf{h}$ & $4 h$ & $5 h$ & $\begin{array}{c}\text { Mea } \\
\mathbf{n}\end{array}$ \\
\hline D. hansenii F3 & 0.04 & 0.03 & 0.03 & 0.03 & 0.03 & 0.03 & 96.0 & 97.0 & 97.0 & 97.0 & 97.0 & 96.8 \\
\hline $\begin{array}{l}\text { C. psychrophila } \\
\text { F6 }\end{array}$ & 0.03 & 0.05 & 0.05 & 0.03 & 0.03 & 0.03 & 97 & 95.0 & 95.0 & 97.0 & 97.0 & 96.2 \\
\hline Mean & 0.05 & 0.04 & 0.03 & 0.02 & 0.02 & & 95 & $\begin{array}{c}95.8 \\
8\end{array}$ & $\begin{array}{c}96.8 \\
8\end{array}$ & 98 & 98 & $\begin{array}{c}96.7 \\
5\end{array}$ \\
\hline $\mathrm{CD}_{0.05}$ & \multicolumn{6}{|c|}{$\begin{array}{l}\mathrm{I}(0.007) \\
\mathrm{T}(0.010)) \\
\mathrm{IxT}(0.022)\end{array}$} & \multicolumn{6}{|c|}{$\begin{array}{l}\mathrm{I}(0.736) \\
\mathrm{T}(0.987) \\
\mathrm{IxT}(2.208)\end{array}$} \\
\hline
\end{tabular}

*Autoaggregation in terms of sedimentation rate

${ }^{\#} \mathrm{OD}_{600}=$ Mean of the results from three separate experiments

$* *$ Autoaggregation $\%=1-\left(\mathrm{A}_{\mathrm{t}=1,2,3,4 \text { and } 5 \mathrm{~h}} / \mathrm{A}_{0 \mathrm{~h}}\right) \times 100$ 
Table.9 Evaluation of Co-aggregation ability of potential probiotic isolates with test indicators

\begin{tabular}{|l|c|c|c|c|}
\hline & \multicolumn{4}{|c|}{ Co aggregation (\%) } \\
\hline Isolates & C. perfringens & L. monocytogenes & B. cereus & Mean \\
\hline D. hansenii F3 & 29.23 & 23.3 & 46.96 & 33.16 \\
\hline C.psychrophila F6 & 45 & 52.72 & 21.31 & 39.67 \\
\hline Mean & 34.15 & 30.70 & 34.23 & \\
\hline
\end{tabular}

\section{Bile salt tolerance}

Resistance to bile salt (oxbile) concentration of screened potential isolates was also studied at different concentrations i.e., $0.3,0.5,1.0$, 1.5 and $2.0 \%$ by viable cell count method during the gastrointestinal transit $\left(\right.$ at $4^{\text {th }} \mathrm{h}$ and $8^{\text {th }} h$ ) as the residence time of food in our body is of about $4 \mathrm{~h}$ Table 6 .

\section{Secondary screening of probiotic yeast isolates}

\section{Cell surface hydrophobicity}

Adherence of cells is usually related to their cell surface characteristics. Cell surface hydrophobicity is a nonspecific interaction between microbial cells and host. The initial interaction may be weak, followed by adhesion processes that are mediated by more specific mechanisms involving cell surface proteins and lipoteichoic acids (Rojas et al., 2002; Ross and Jonsson, 2002) (Table 7).

\section{Autoaggregation}

Autoaggregation ability determines the capacity of the probiotic strain to interact with itself in a nonspecific way. Aggregation between cells of the same strain (autoaggregation) or between different species and strains (coaggregation) is of considerable importance and is one of the key factors to check the ability of the probiotic strain to adhere to the oral cavity and gastrointestinal tract, where probiotics are to be active and provide their beneficial effects (Nikolic et al., 2010) (Table 8).
Autoaggregation assay was performed as described by Del Re et al., (2000)

\section{Co-aggregation (Del Re et al., 2000)}

Probiotic and pathogenic microorganisms have been reported to form joint aggregate and the process is known as coaggregation (Surono, 2004). Probiotics are able to achieve coaggregation with pathogenic microorganisms and in turn effectively inhibit and kill them by secreting antimicrobial compounds that act directly on the cells of pathogenic bacetria (Bao et al., 2010) (Table 89).

In the present study, 18 yeast strains were isolated, and characterized from sidra based on their morphological and biochemical characteristics and among them, two i.e., F6 and F3 were potential producers of antimicrobial substances against various food borne pathogens. The selected strains exhibited outstanding production of antimicrobial activity. The above mentioned results support the use of native probiotic yeasts strains in starter cultures or as preservative agents which can be achieved by inhibition of contamination causing food borne pathogens.

\section{References}

Cocolin, L, Bautista-Gallego, J., ArroyoLópez, F.N., Rantsiou, K., JiménezDíaz, R., GarridoFernández, A., 2013. Screening of lactic acid bacteria isolated from fermented

Conway PL, Gorbach SL and Goldin 
BR.1987. Survival of lactic acid bacteria in the human stomach and adhesion to intestinal cells. Journal of Dairy Science 70, 1-12

Del Re B, Sgorbati B, Miglioli $M$ and Palenzona D. 2000. Adhesion, autoaggregation and hydrophobicity of 13 strains of Bifidobacterium longum. Letters in Applied Microbiology 31: 438-442

Kurtzman, C.P., Robnett, C.J., 1998. Identification and phylogeny of ascomycetous yeasts from analysis of nuclear large subunit (26S) ribosomal DNA partial sequences. Antonie Van Leeuwe

Mishra V and Prasad DN. 2005. Application of in vitro methods for selection of Lactobacillus casei strains as potential probiotics. International Journal of Food Microbiology 103:109-115.

Salampessy J, Kailasapathy N. Thapa Fermented fish products J.P. Tamang $\mathrm{J}$ P, Kailasapathy, K. (Eds.), Fermented foods and beverages of the world, CRC Press, Taylor \& Francis Group, New
York (NY) (2010), pp. 289-307

Tamang J, P. Himalayan fermented foods: microbiology, nutrition, and ethnic values CRC Press, Taylor \& Francis Group, New York (NY) (2010)

Tamang JP. 2010. Himalayan Fermented Foods: Microbiology, Nutrition, and Ethnic 257 Values, CRC Press, Taylor \& Francis Group, New York.

Tamang P. Brief note on fish diversity of Teesta and Rangit river system in Sikkim and their conservation A report of State Council of Science and Technology for Sikkim, Gangtok (2002), p. 4

Walker DK and Gilliland SE. 1993. Relationship among bile tolerance, bile salt deconjugation, and assimilation of cholesterol by Lactobacillus acidophilus. Journal of Dairy Science 45:178-182.

Whittenbury, R. 1964. Hydrogen peroxide formation and catalase activity in the lactic acid bacteria. Journal of General Microbiology 35, 13e26.

\section{How to cite this article:}

Jasveen Bajwa and Nivedita Sharma. 2018. Evaluation of Probiotic Properties of Yeasts Isolated from Sidra - An Ethnic Fermented Fish Product of North East India. Int.J.Curr.Microbiol.App.Sci. 7(02): 2632-2643. doi: https://doi.org/10.20546/ijcmas.2018.702.320 